\title{
Determinación de índice de infestación por Demodex spp, en pacientes con blefaritis crónica y en pacientes sin otra patología ocular
}

\author{
Nancy Rivera, Priscila Molina y Andrea Torres
}

Universidad de Concepción, Concepción, Chile (NR) Alumna Carrera de Tecnología Médica, mención oftalmología (PM).

Hospital Guillermo Grant Benavente, Concepción, Chile (AT).

Financiamiento: Recursos propios de la Carrera de Tecnología Médica, Universidad de Concepción. Los autores declaran no tener conflictos de interés.

Recibido: 10 de agosto de 2012 Aceptado: 6 de agosto de 2013

Correspondencia a: Nancy Rivera Fuentes nrivera@udec.cl

\section{Measurement of the index of infection by Demodex spp in patients with chronic blepharitis and in patients without other ocular diseases}

Background: Infestation by Demodex spp. in the palpebral edge follicles in humans is common. However, these mites are not routinely diagnosed in patients with or without ocular pathologies in Chile and their relevance is unclear. One of the eye diseases most related to infestation by Demodex spp. is blepharitis, a chronic inflammation of the eyelid margin with intermittent exacerbations, which is very common in ophthalmic practice. Its management is prolonged treatment, which is often ineffective, leading to relapses and frustration of patient and treating physician. Blepharitis can be typed by its etiology into various types, one of them is caused by Demodex folliculorum and another species, D. brevis. Objective: The overall objective was to detect the presence and estimate the rate of infestation of Demodex spp. in healthy subjects and in patients with ocular pathology such as blepharitis, bacterial conjunctivitis, chalazion, and stye. Patients and Methods: Samples of tabs from both lower eyelids of ophthalmologically healthy patients (23) and patients with ocular pathologies (9) were mounted in immersion oil as described in literature, then visualized with a 10x objective and confirmed with 40x objective. Results. Detection rates of eggs, nymphs or adults of Demodex spp. in patients with and without ophthalmological problems were above and below 0.5 mites per tab, respectively. $D$. folliculorum was the species most frequently found. Discussion: The results are consistent with the international literature on both the rate of infestation as the predominant species. Conclusion: This paper is the first study in Chile on this subject and represents a significant contribution to ophthalmic clinical diagnosis and treatment of patients with this disease.

Key words: Demodex spp., blepharitis, infestation index

Palabras clave: Demodex, blefaritis, indice de infestación.

\section{Introducción}

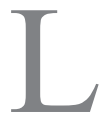
a blefaritis es una enfermedad muy común en la práctica oftalmológica. Normalmente es de curso crónico, con exacerbaciones intermitentes de los síntomas ${ }^{1,2}$. Consiste en la inflamación crónica del borde palpebral $^{3}$; su origen puede ser multifactorial por lo que hay que tratarla de diversas maneras, más aún si hay patologías asociadas, como la disfunción de la película lagrimal, infecciones virales, dermatitis seborreica alérgica, conjuntivitis o queratitis ${ }^{1}$. Si no se detiene el proceso, se produce un empeoramiento progresivo, con inflamación dolorosa, pudiendo aparecer incluso orzuelos y chalazión. Clínicamente puede ser asintomática ${ }^{4}$ y constituir un enigma diagnóstico y terapéutico; de hecho, su manejo suele llevar mucho tiempo frecuentemente ineficaz, con múltiples recaídas que terminan desmoralizando al paciente y al médico que las trata ${ }^{5}$. De acuerdo a la etiología, se describen varios tipos de blefaritis ${ }^{6}$. Su causa es una disfunción de las glándulas sebáceas que motiva una acumulación de secreciones, y su posterior sobreinfección; en reiteradas ocasiones pasa desapercibida por el médico especialista ${ }^{2,7}$. Para algunos autores como Eva Vico, Antonio Vallejo y José Benítez del Castillo, en Gran Bretaña las blefaritis constituyen el $71 \%$ de los casos que acudían al médico general por enfermedad ocular y, en España el 66\% de los pacientes que acuden a consulta oftalmología general la padecen ${ }^{5}$. Según su localización puede ser anterior, si están hacia la parte libre del parpado, o posterior si se acercan al ojo ${ }^{5,7}$ o mixta; según el microorganismo involucrado, puede clasificarse en bacteriana, mixta o viral ${ }^{2,4,8}$. Lydia Griffths clasifica la blefaritis en: escamosa, caracterizada por la formación de escamas entre las pestañas, las cuales muchas veces caen al interior de los ojos produciendo sensación de cuerpo extraño, ojos rojos; alérgica, que en su mayoría corresponde a alergia a productos cosméticos, e incluso pueden ser causadas por los medicamentos utilizados para los ojos, como colirios y 
pomadas y blefaritis asociadas a Demodex folliculorum ${ }^{7}$, siendo esta última motivo de esta publicación.

Demodex (del griego: demos = grasa; dex = carcoma) pertenece al phylum artrópoda, clase Arácnida, orden Acarina, (superfamilia: Demodicodoidea) y su infestación en el humano se le conoce como demodicidosis o demodecosis $^{9}$. Estos ácaros se concentran en sitios corporales donde las glándulas sebáceas son numerosas y la producción de grasa es abundante. Se han encontrado dos subespecies, longus y brevis. Demodex folliculorum (longus) parasita los folículos pilosos, las glándulas sebáceas y las pestañas del hombre, y puede encontrarse en solitario o en grupos en la apertura folicular; $D$. brevis se encuentra en la glándula sebácea y como individuo único ${ }^{3}$. Ambos se encuentran en todas las razas humanas, sin preferencia de $\operatorname{sexo}^{10-14}$. Demodex spp. es llamado también ácaro del folículo piloso. Es un parásito frecuente y extendido, presenta dimorfismo sexual y en su ciclo es posible observar huevos, larvas, protoninfa ninfas, macho y hembra adulta ${ }^{15}$.

El rol patógeno de Demodex spp., aún no ha sido determinado. Se cree que juega un papel patogénico cuando se multiplica y (raramente) entra a la dermis, pero en otros países se está considerando como causante o factor que contribuye al desarrollo de enfermedades oculares y/o dérmicas; muchos autores lo señalan como un parásito oportunista, o como un vector portador de microorganismos ${ }^{2,13,25}$.

La forma de contagio es por contacto directo ${ }^{12,15}$, es así como puede ser encontrado en personas de todas las edades excepto en recién nacidos. Las formas infestantes son todos los estadios larvarios y adultos del parásito. $\mathrm{Su}$ distribución es cosmopolita. Algunos autores plantean que Demodex sp podría actuar como un vector de hongos y bacterias, en particular, de Bacillus oleronius, Staphylococcus aureus y Streptococcus $\mathrm{spp}^{2,25}$. Un dato a favor que sirve para relacionar al ácaro como vector en la infección estafilocóccica es el hecho de haber encontrado bacterias como B. oleronius y Staphylococcus epidermidis (menos frecuentemente) en el interior de ejemplares de D. folliculorum. También hay quienes manifiestan que pueden vivir en simbiosis con el hombre ${ }^{1,9,16,17}$.

La prevalencia de Demodex spp reportada en piel de humanos, de diferentes poblaciones, pueden variar desde 0 a $100 \%$. Según un estudio, esto se considera como resultado de la edad de los grupos estudiados y al método de recolección de las muestras para la observación del ácaro. La prevalencia de Demodex spp., aumenta con la edad, quizás atribuyendo esto a que los ancianos producen una mayor secreción sebácea y ensanchamiento de la apertura folicular propia de la esta etapa de la vida ${ }^{3}$. En niños y adolescentes la infestación es inhabitual, presumiblemente a que durante la infancia hay una baja producción de sebo ${ }^{13}$.

Según un estudio realizado en el Hospital de Clínicas ${ }^{4}$,
Paraguay, se observó una frecuencia de 54\% de Demodex spp. en pacientes con blefaritis crónica, resultado que coincide con otros estudios reportados y demuestra el importante papel que juega en la patogenia de la blefaritis crónica ${ }^{16}$.

Un altísimo porcentaje de las blefaritis crónicas tiene como etiología a este parásito de los anexos externos del ojo. El diagnóstico diferencial se realiza con el examen de lámpara de hendidura de las formaciones vesiculosas que confirmará la presencia del parásito ${ }^{14}$. Además se realiza el examen microscópico, en busca de la presencia del ácaro en las pestañas.

Demodex spp. está involucrado en cuadros oculares como chalazión, orzuelo interno crónico ${ }^{7}$ y síndrome de ojo seco ${ }^{2,10,19,20}$. La inflamación producida puede extenderse hacia la conjuntiva, produciendo una conjuntivitis, e incluso causar lesiones corneales ${ }^{22}$.

La infestación por Demodex spp. en los folículos del borde palpebral en humanos es frecuente; no obstante, en Chile no hay datos publicados. El presente estudio tiene como objetivo general detectar la presencia y conocer el índice de infestación de Demodex spp. en pacientes sanos y en aquellos que presenten alguna patología ocular como blefaritis crónica, conjuntivitis crónica, chalazión u orzuelo, como una forma de contribuir al diagnóstico clínico oftalmológico.

En este estudio se plantearon las siguientes hipótesis:

- Demodex spp. se encuentra en mayor cantidad en el folículo pilosebáceo en pacientes con blefaritis crónica que en pacientes que no presentan esta patología ocular.

- Demodex folliculorum es la especie predominante, tanto en pacientes con blefaritis crónica como en aquellos que no presentan esta patología ni otras de la esfera oftalmológica.

- El índice de infestación de Demodex spp. aumenta en pacientes de mayor edad.

Los objetivos específicos fueron los siguientes:

- Detectar la presencia de Demodex spp. en pacientes con patologías oculares, como blefaritis, y en pacientes sin éstas.

- Determinar el índice de infestación con Demodex spp. en pacientes sanos y en aquellos que presenten alguna patología ocular palpebral como blefaritis crónica, conjuntivitis crónica, chalazión u otra.

- Establecer la especie de ácaro predominante en la población estudiada.

\section{Pacientes y Métodos}

Estudio observacional, de corte transversal.

\section{Universo}

Pacientes ambulatorios que voluntariamente aceptaron la extracción de pestañas para toma de muestra, previo 
a repetición, se pudo observar muchos ejemplares adultos de D. folliculorum en una sola pestaña (Figura 3).

En la Tabla 1 y en la Figura 4 podemos observar que la presencia de Demodex spp. fue mayor en el grupo de pacientes con patologías oculares (44\%), a diferencia del grupo control (9\%) y que los pacientes del grupo control pertenecían, mayoritariamente, al rango de edad de 21 a 30 años; en cambio, en los pacientes con patologías oculares las edades fluctuaron entre 11 y 70 años y la mayor frecuencia se dio en pacientes en el rango 51 a 60 años.

Fue posible observar que el total de pacientes en cada grupo presentó un índice de infestación que está por debajo del índice establecido por la literatura internacional.

En los pacientes que se encontró la presencia de Demodex spp, se pudo observar que tanto en el grupo control como en el grupo patológico, predominó $D$. folliculorum, lo cual se puede observar en las Tablas $4 \mathrm{y}$ 5 , respectivamente.

En un paciente se encontró la presencia simultánea de D. folliculorum y D. brevis, siendo mayor el número de ácaros de la especie folliculorum en el total de las pestañas observadas. El grupo etario con patología palpebral, entre 51 y 60, tuvo una mayor prevalencia de Demodex spp. lo cual es significativo para esta muestra, siendo la especie predominante $D$. folliculorum (Tabla 4).

\section{Discusión}

Los resultados muestran un patrón, semejante al obtenido en las revisiones bibliográficas consultadas. Los datos de esta investigación, aunque no es representativa de
Tabla 1. Presencia de Demodex spp., según rango etario, en el grupo control y pacientes con patologías palpebrales

\begin{tabular}{|ccccc|}
$\begin{array}{c}\text { Rango de edad } \\
\text { (años) }\end{array}$ & $\mathbf{n}$ & $\begin{array}{c}\text { Grupo control } \\
\text { Demodex spp (+) }\end{array}$ & $\begin{array}{r}\text { Pacientes con patologías palpebrales } \\
\mathbf{n}\end{array}$ & $\begin{array}{r}\text { Demodex spp (+) } \\
11-20\end{array}$ \\
$21-30$ & 16 & 0 & 2 & 0 \\
$31-40$ & 2 & 1 & 0 & 0 \\
$41-50$ & 2 & 0 & 2 & 1 \\
$51-60$ & 2 & 0 & 2 & 2 \\
$61-70$ & 0 & 0 & 1 & 0 \\
Total & 23 & 2 & 9 & 4 \\
\hline
\end{tabular}

Tabla 2. Índice de infestación promedio según grupo etario en ambos grupos

\begin{tabular}{|c|c|c|c|c|}
\hline \multirow[t]{2}{*}{$\begin{array}{l}\text { Rango de edad } \\
\text { (años) }\end{array}$} & \multicolumn{2}{|c|}{ Grupo control } & \multicolumn{2}{|c|}{$\begin{array}{l}\text { Grupo patología } \\
\text { palpebral }\end{array}$} \\
\hline & $\begin{array}{l}<0,5 \\
(a / p)\end{array}$ & $\begin{array}{l}\geq 0,5 \\
(\mathrm{a} / \mathrm{p})\end{array}$ & $\begin{array}{l}<0,5 \\
(a / p)\end{array}$ & $\begin{array}{l}\geq 0,5 \\
(\mathrm{a} / \mathrm{p})\end{array}$ \\
\hline $11-20$ & 0 & 0 & 0 & 0 \\
\hline $21-30$ & 0,25 & 0 & 0 & 0 \\
\hline $31-40$ & 0 & 0,5 & 0 & 0 \\
\hline $41-50$ & 0 & 0 & 0,25 & 0 \\
\hline $51-60$ & 0 & 0 & 0 & 1,625 \\
\hline $61-70$ & 0 & 0 & 0 & 0 \\
\hline
\end{tabular}

a/p: ácaros por pestaña.

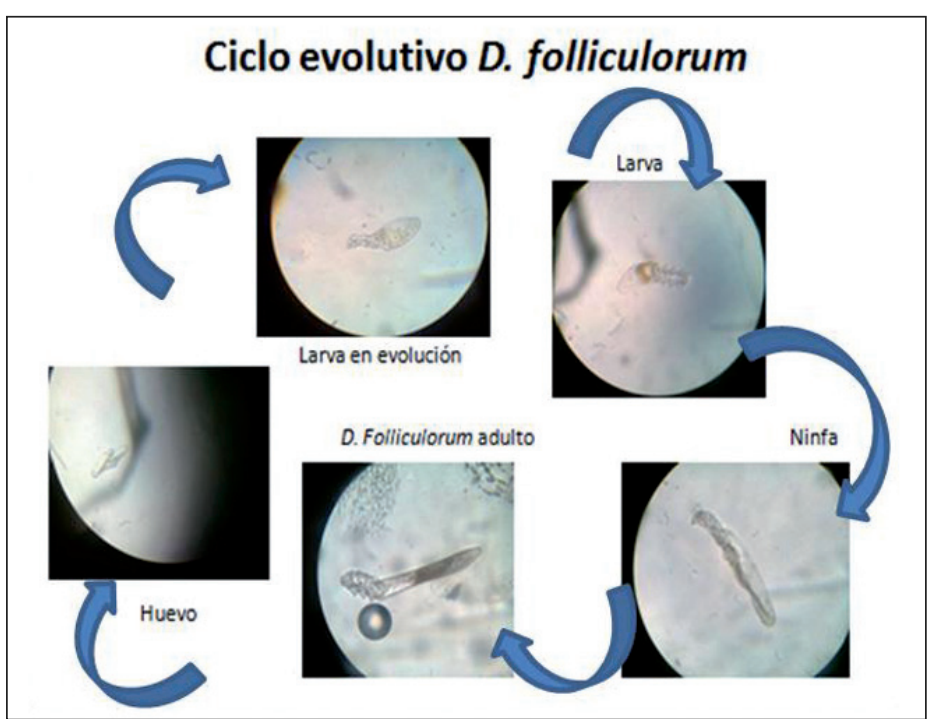

Figura 1. Ciclo evolutivo en una muestra de un paciente control dejada $48 \mathrm{~h}$ en aceite de inmersión.

$\begin{aligned} & \text { Tabla 3. Cuadro comparativo del índice de infestación promedio de } \\ & \text { ambos grupos }\end{aligned}$
$\begin{array}{lcc}\text { Grupo } & \begin{array}{c}\text { Índice de infestación } \\ \text { total pacientes }(\mathrm{a} / \mathrm{p})\end{array} & \begin{array}{c}\text { Índice de infestación } \\ \text { promedio positivos }(\mathrm{a} / \mathrm{p})\end{array} \\ \text { Control } & 0,03 & \mathbf{0 , 3 7} \\ \text { Patología palpebral } & 0,44 & \mathbf{1 , 0 0}\end{array}$
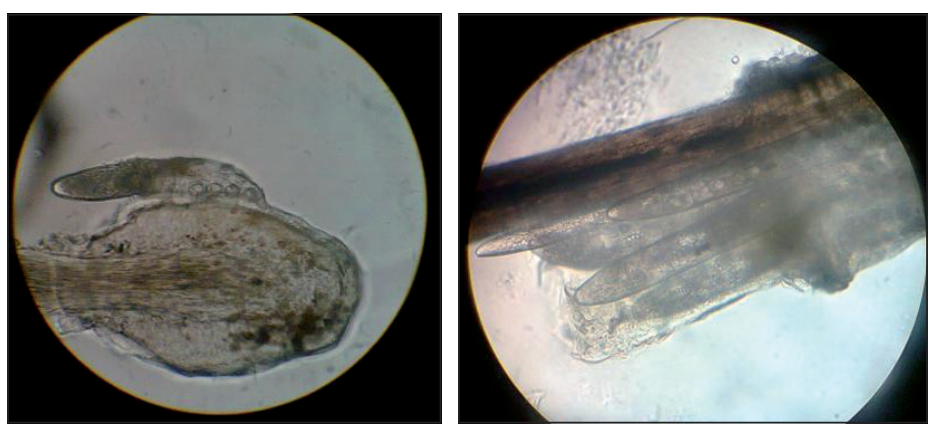

Figura 2. Demodex folliculorum, inmerso en aceite de inmersión (40x), encontrados en uno de los pacientes. 
Figura 3. Demodex folliculorum, en aceite de inmersión (observado con objetivo 40x), encontrado en un paciente varón, de 58 años, con chalazión a repetición.
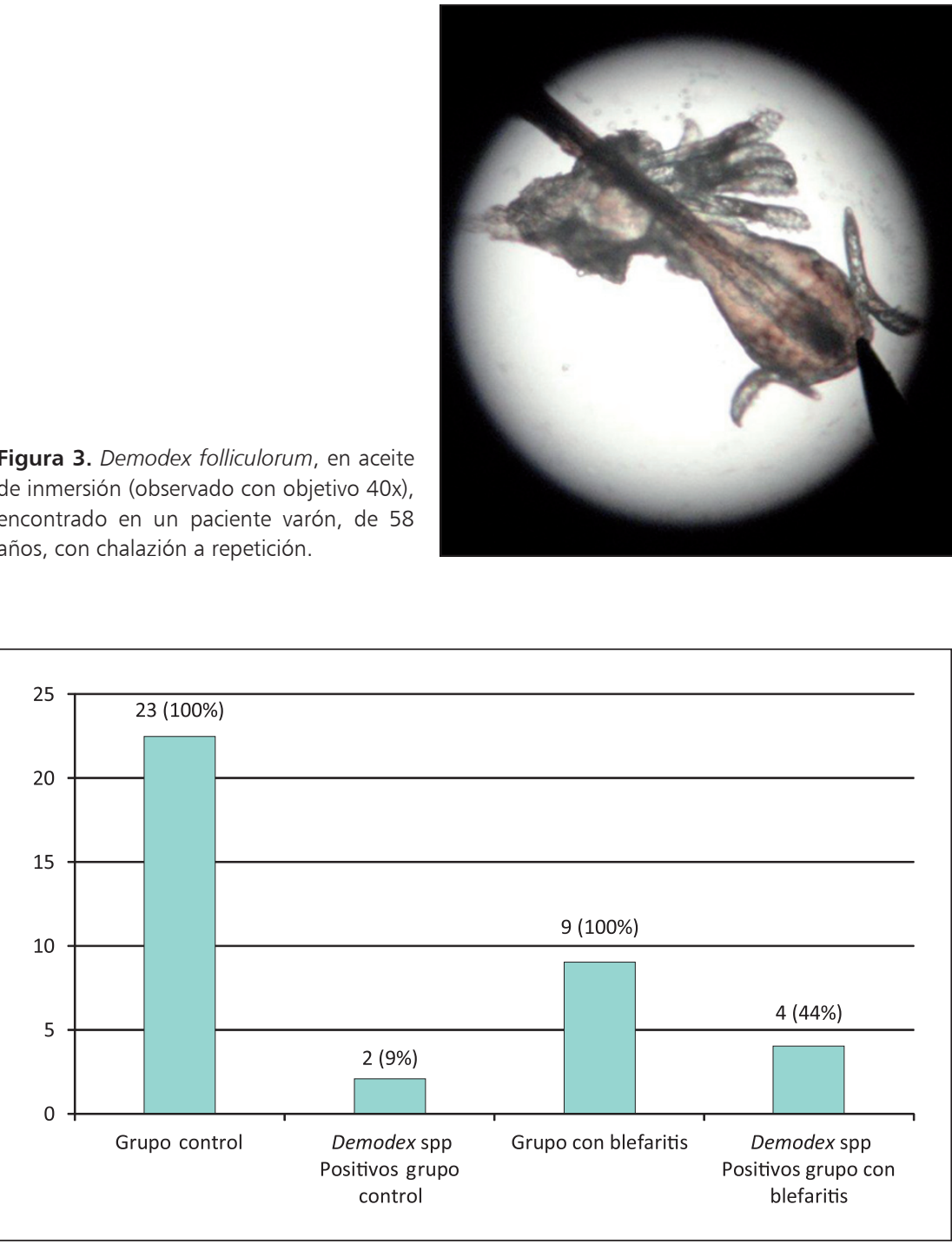

Figura 4. Prevalencia de Demodex spp. en el grupo con blefaritis versus grupo control.

Tabla 4. Presencia de $D$. folliculorum vs $D$. brevis en grupo control y grupo con blefaritis (Sólo considerando especies adultas (sin contar huevos)

\begin{tabular}{|c|c|c|c|c|}
\hline \multirow[t]{2}{*}{$\begin{array}{l}\text { Rango de edad } \\
\text { (años) }\end{array}$} & \multicolumn{2}{|c|}{$\begin{array}{c}\text { Demodex } \\
\text { Grupo control }\end{array}$} & \multicolumn{2}{|c|}{$\begin{array}{c}\text { Demodex } \\
\text { Grupo con patología palpebral }\end{array}$} \\
\hline & $\begin{array}{l}\text { D. folliculorum } \\
\mathrm{n}\end{array}$ & $\begin{array}{l}\text { D. brevis } \\
\mathrm{n}\end{array}$ & $\begin{array}{l}\text { D. folliculorum } \\
\mathrm{n}\end{array}$ & $\begin{array}{l}\text { D. brevis } \\
\mathrm{n}\end{array}$ \\
\hline $10-20$ & 0 & 0 & 0 & 0 \\
\hline $21-30$ & 1 & 0 & 2 & 0 \\
\hline $31-40$ & 2 & 1 & 0 & 0 \\
\hline $41-50$ & 0 & 0 & 0 & 0 \\
\hline $51-60$ & 0 & 0 & 10 & 4 \\
\hline $61-70$ & 0 & 0 & 0 & 0 \\
\hline Total & 3 & 1 & 12 & 4 \\
\hline
\end{tabular}

la población general, deja en evidencia que Demodex spp se encuentra tanto en pacientes controles como en aquellos con patologías oculares; no obstante, se observan diferencias estadísticamente significativas, bajo prueba de $\chi^{2}$ un $99 \%$ de confianza $(\mathrm{p}<0,01)$ que demuestran que tanto la infestación como el índice de infestación, es mayor en los pacientes con blefaritis y/o en las patologías oculares ya mencionadas, que en el grupo control (Tablas 1 y 3 ).

En las muestras estudiadas, se encontró mayor presencia de $D$. folliculorum, en ambos grupos, lo que coincide, con lo que se ha encontrado en la revisión bibliográfica $^{21-23}$.

Como ya fue señalado anteriormente, en una de las muestras del grupo control se encontró ambas especies; por motivo de tiempo fueron revisadas y fotografíadas con tardanza (48 h), encontrándose la visualización del ciclo completo de $D$. folliculorum y larvas de $D$. brevis en evolución. Al respecto, Román Pereira y cols., señalan en su investigación la mayor sobrevida de Demodex spp en aceite de inmersión; en refrigeración sobreviven tan sólo $15 \mathrm{~min}$, y si se conservan bajo condiciones ordinarias mueren en pocas horas.

Se pudo observar que, según el índice de infestación establecido en el grupo control, los resultados por grupo etario estarían dentro de lo que en la literatura médica internacional se describe como "normal"; no obstante, en el rango de edad 31 a 40 años estaría en el límite. Es notorio el aumento por sobre el índice de infestación "normal", en los pacientes del grupo patológico con rango de edad entre 51 y 60 años.

Cabe resaltar la sencillez del método diagnóstico de la blefaritis por Demodex spp, además de ser de bajo costo. No obstante, se requiere de un profesional con experiencia y adiestrado en la detección y diagnóstico del ácaro, por lo que se aconseja la búsqueda del parásito cuando la manifestación clínica así lo requiera.

Al calcular el índice de infestación promedio en ambos grupos, se encontró una gran diferencia, lo cual confirma la hipótesis de que los pacientes con las patologías oculares ya mencionadas presentan un alto grado de infestación.

En el grupo control se encontró $D$. brevis en un solo paciente y en menor cantidad que $D$. folliculorum. En el grupo con patología palpebral se encontró $D$. brevis en un paciente con diagnóstico de orzuelo y, en el paciente que presentó el mayor índice de infestación $(2,25 \mathrm{a} / \mathrm{p})$ sucedió lo mismo, siendo la especie más frecuente $D$. folliculorum, lo cual valida la hipótesis de que $D$. folliculorum es la especie predominante. Ante estos resultados y, discutiendo entre los investigadores, queda la duda con respecto a la presencia de D. brevis, ya que por su localización (glándula de Meibomio), para su búsqueda no sería la extracción de pestañas lo más adecuado sino, recoger otro tipo de muestra, extraída por un oftalmólogo, sobre todo en aquellos pacientes en que 
se sospecha la presencia del ácaros pero, los resultados son negativos.

En cuanto a la capacidad patógena de Demodex spp. en afecciones oculares, Liu J, Sheha H y SC Tseng, 2010²5, describen que han sido propuestos tres mecanismos de acción para explicar su rol patógeno:

- Daño directo. Especialmente atribuible a D. folliculorum, puesto que los ácaros consumen las células epiteliales en el folículo piloso que resulta en la distensión folicular, pudiendo contribuir a la formación de pestañas que se caen o están mal dirigidas. Las microabrasiones causadas por las garras del ácaro pueden provocar hiperplasia epitelial e hiperqueratinización reactiva alrededor de la base de las pestañas, con la consiguiente formación de la caspa cilíndrica, lo cual se observa regularmente en los pacientes afectados. Por otra parte, D. brevis puede bloquear mecánicamente los orificios de las glándulas de Meibomio, dando lugar a disfunción de estas glándulas.

- Demodex spp. puede causar blefaritis al actuar como vector de bacterias en su superficie, incluyendo estreptococos y estafilococos los cuales producen proteínas que actúan como superantigenos gatillando una fuerte respuesta inflamatoria.

- Reacciones de hipersensibilidad. Las proteínas (superantigenos) dentro de los ácaros Demodex y sus desechos, pueden provocar respuestas inflamatorias del hospedero a través de una hipersensibilidad retardada o una respuesta inmune innata ${ }^{25}$.

Por todo lo anterior, no se puede negar el rol patogénico de estos ácaros, lo que hace necesario su detección y tratamiento oportunos.

Acerca del tratamiento de afecciones oculares donde se ha encontrado presencia de ácaros, la bibliografía revisada señala que, el primer paso a realizar, es la eliminación de las formaciones vesiculosas de las pestañas y de este modo eliminar el parásito. Un método eficaz consiste en instilar una gota de anestésico en el ojo y, con un algodón mojado con éter se masajean fuertemente los bordes palpebrales una vez por semana. Esto permite limpiar las excretas del parásito y así las colas del ácaro comienzan a salir de los folículos pudiéndose ver en la lámpara de hendidura. Esto sucedería debido al efecto tóxico que produce en el ácaro. Luego, aplicación de pomada de óxido de amarillo de mercurio al $2 \%$, durante 7 días. El tratamiento se repite a los 15 días para erradicar la visualización microscópica del parásito vivo ${ }^{2,14}$.

Otro estudio refiere, que una sola dosis de ivermectina oral, de 100 a $200 \mu \mathrm{g} / \mathrm{kg}$ es suficiente contra Demodex spp $^{13}$. Holzchuh FG y cols., 2011, en consideración al ciclo del parasito, recomiendan repetir el tratamiento a los siete días, indicando que se reduce notablemente la cantidad de ácaros post-tratamiento y que ivermectina oral es bien tolerada por los pacientes ${ }^{26}$. Ivermectina se comercializa en Chile y ha sido utilizada por uno de los autores (A. Torres), para tratar pacientes con patologías oculares como la blefaritis asociada a la presencia de Demodex spp. (Comunicación personal).

\section{Conclusiones}

Al encontrar la presencia de Demodex spp. en ambos grupos, se reafirma que éste es un parásito que habita normalmente en humanos.

El índice de infestación encontrado en ambos grupos concuerda con lo referido en la bibliografía, siendo el índice de infestación promedio $0,37 \mathrm{a} / \mathrm{p}$ en el grupo control y de 1,0 a/p en el otro grupo, validando la afirmación de que un índice elevado está en estrecha relación en el desarrollo de las patologías relacionadas.

La especie más frecuente en ambos grupos fue $D$. folliculorum, con la salvedad que se requiere otro tipo de muestra para el diagnóstico de $D$. brevis por la ubicación que presenta el parásito.

Demodex spp. se encontró con mayor frecuencia en pacientes de los rangos etarios 21 a 40 y, 51 a 60 años (grupo control y grupo patológico, respectivamente), por lo que se encontraría en los pacientes que presentan mayor edad, siendo esto un dato de importancia, sumado al hecho que en pacientes añosos, los síntomas y signos relacionados a patología palpebral van en aumento de acuerdo aumenta la edad.

El método utilizado resultó apropiado para realizar el diagnóstico de $D$. folliculorum siendo un método de diagnóstico sencillo, rápido, seguro y económico tanto para el paciente, como para el profesional especializado en la detección de Demodex spp.

Finalmente, se concluye que es un examen que debiese realizarse de rutina en pacientes con las patologías oculares mencionadas y que no responden a tratamiento.

\section{Proyecciones}

Para obtener resultados representativos de la población es necesario aumentar el número de pacientes, en especial de los pacientes oftalmológicos, lo que permitirá extrapolar los resultados y obtener estadísticos que permitan establecer si las diferencias encontradas son estadísticamente significativas.

Se recomienda realizar examen de búsqueda de ácaros Demodex spp. en pestañas, en aquellos pacientes que presenten signos de blefaritis crónica resistente a tratamientos convencionales, chalazión u orzuelos a repetición y en conjuntivitis bacteriana reiterativa.

Agradecimientos. A todos los pacientes que voluntariamente aceptaron participar en este estudio. Al Dr. Sergio 
Micco. Laboratorio Lincoyán de Concepción. A las autoridades de la Carrera de Tecnología Médica, Facultad de Medicina Universidad de Concepción por el apoyo brindado a esta investigación y, a las autoridades de Hospital Guillermo Grant Benavente por haber visado el Consentimiento Informado que se utilizó en este estudio y haber permitido realizar la toma de muestras a algunos pacientes del Servicio de Oftalmología del mencionado hospital. A Patricia Huerta San Martín por el apoyo estadístico de este estudio.

\section{Resumen}

Introducción: La infestación por Demodex spp. en los folículos del borde palpebral en humanos es frecuente; no obstante, en Chile no se diagnostica de rutina la presencia de estos ácaros en pacientes sin o con patologías oculares por lo cual no se conocen aspectos de esta parasitosis. Una de las patologías oculares que más se relaciona con infestación por Demodex spp. es la blefaritis, enfermedad muy común en la práctica oftalmológica, que cursa con inflamación crónica del borde palpebral, con exacerbaciones intermitentes de los síntomas. Su manejo suele llevar mucho tiempo frecuentemente ineficaz, con múltiples recaídas que terminan desmoralizando al paciente $\mathrm{y}$, al médico que las trata. De acuerdo a la etiología, se carac- terizan varios tipos de blefaritis y una de ellas es asociada a Demodex folliculorum, existiendo también la especie $D$. brevis. Objetivo: Detectar la presencia y calcular el índice de infestación de Demodex spp. en pacientes sanos y en pacientes con alguna patología ocular como blefaritis crónica, conjuntivitis bacteriana, chalazión y orzuelo. Pacientes y Métodos: Se tomaron muestras de pestañas desde el párpado inferior de ambos ojos en pacientes oftalmológicamente $\operatorname{sanos}^{23}$ y pacientes con patologías oculares $^{9}$ las que fueron montadas en aceite de inmersión según técnica descrita en la literatura, visualizadas con objetivo 10x y confirmadas con objetivo 40x. Resultados: Se encontró la presencia de huevos, ninfas y ejemplares adultos de $D$. folliculorum y $D$. brevis, tanto en pacientes normales como en pacientes oftalmológicos siendo el índice de infestación menor a 0,5 ácaros por pestaña en los pacientes sanos y mayor o igual a 0,5 en pacientes oftalmológicos. La especie más frecuentemente encontrada fue D. folliculorum. Discusión: Los resultados encontrados coinciden con la literatura internacional tanto en el índice de infestación como en la especie predominante. Conclusión: Este trabajo constituye el primero realizado en Chile en esta temática, de acuerdo a la literatura revisada, lo cual constituye un gran aporte al diagnóstico clínico oftalmológico con implicancias en el tratamiento de estos pacientes.

\section{Referencias bibliográficas}

1.- Rodríguez A E, Ferrer C, Alió J L. Demodex y blefaritis crónica. Arch Soc Esp Oftalmol 2005; 80 (11). Disponible en: http://scielo.isciii. es/scielo.php?script $=$ sci_arttext\&pid $=$ S0365$66912005001100004 \& \operatorname{lng}=$ en \&nrm $=$ iso\&tlng= en [accedido el 15 de diciembre de 2011).

2.- Román Pereira Y, Verona Ugando L, Hernández Conde M, Díaz Díaz Y. Panorámica actual en la blefaritis por Demodex folliculorum. Revista Misión Milagro 2009; 3 (3) Ciudad de La Habana. Disponible en: http://www. misionmilagro.sld.cu/vol3no3/pdf/rev3303.pdf [accedido el 24 de diciembre de 2011].

3.- Fernández Pérez S R, Dios Lorente J A de, Peña Sisto L, García Espinosa S M, León Leal M. Causas más frecuentes de consulta oftalmológica. [Artículo en línea]. MEDISAN 2009: 13 (3). Disponible en http://bvs.sld. cu/revistas/san/vol13_3_09/san10308.pdf [accedido el 26 de diciembre de 2011].

4.- Perdomo J, Acosta K, Palacios S, Carrero M, González E. Homeopatía y Oftalmología: las blefaritis. Rev Cubana Med Milit 2007; 36 (3). Disponible en: http://bvs.sld.cu/ revistas/mil/vol36_3_07/mil07307.html [accedido el 18 de diciembre de 2011].

5.- Vico E, Vallejo A, Benítez J. Blefaritis e higiene palpebral Unidad de Superficie e Inflamación Ocular. Hospital Clínico San Carlos (Madrid): pp 2. Disponible en http://www.laboratoriosthea.com/archivos/ publicaciones/00106.pdf [accedido el 24 de diciembre de 2011].

6.- Godínez A, Medina C, Velázquez L, García C, González S. Prevalencia de los ácaros Demodex folliculorum y Demodex brevis en una población mexicana. Medicina Universitaria 2004; 6 (23): 96-100. Disponible en: http://drjair.com/PDF/ MEDICO/ENSAYOS/prevalencia_acaros.pdf [accedido el 16 de diciembre de 2011].

7.- Griffiths L. La Blefaritis: Un "círculo vicioso" (Entrega I). Jueves, 07 de julio de 2011. Disponible en: http://www.tucontactomedico. com/index.php?view $=$ article $\&$ catid $=93 \% 3$ Ade-los-ojos\&id=417\%3Ala-blefaritis-un-circulo-vicioso-entrega-i-autor-dralydia-griffiths \& format $=$ pdf\&option $=$ com content\&Itemid=93 [accedido el 18 de diciembre de 2011].

8.- Betancurt C, Agudelo L, Durán P, Muñoz M. Clínica y microbiología de blefaritis en pacientes atendidos en la Fundación Universitaria del Área Andina Pereira. 2003. Red de Rev Científicas de América Latina, el Caribe, España y Portugal. Investigaciones Andina 2003; 7: 20-5. Disponible en: http:// redalyc.uaemex.mx/src/inicio/ArtPdfRed. jsp?iCve $=239017952005$ [accedido el 18 de diciembre de 2011]

9.- Sedeño I, Novoa E, Padrón V, García F, San Martín R. Blefaritis por Demodex folliculorum. Diagnóstico y tratamiento. Rev Cubana Oftalmol 2006; 19 (1): 1-5. Disponible en: http://bvs.sld.cu/revistas/oft/ vol19_1_06/oft03106.pdf [accedido el 18 de diciembre de 2011].

10.- Chin-Wong J L, Niño-Pecina A, Ruiz-Quintero N, Naranjo-Tackman R. Prevalencia de demodecosis en pacientes con blefaroconjuntivitis crónica multitratada. Rev Mex Oftalmol 2006; 80 (2): 61-3. Disponible en: http:/www.medigraphic. com/pdfs/revmexoft/rmo-2006/rmo062b.pdf [accedido el 20 de diciembre de 2011].

11.- Romero D. Demodex spp. Los ácaros cara: Los ácaros son parásitos comunes del folículo de la piel del rostro humano. 29 de agosto de 2007. Disponible en: http://translate.google.cl/transl ate?hl=es\&langpair=en|es\&u=http://rosemarydrisdelle.suite101.com/demodex-spp-facemites-a30043 [accedido el 26 de diciembre de 2011].

12.- Serrano P. Dermatosis inducidas por Demodex folliculorum. PIEL. 2011; 287: 5. Disponible en: http:/www.elsevier.es/sites/default/files/ elsevier/eop/S0213-9251\%2811\%2900190-0. pdf [accedido el 26 de diciembre de 2011].

13.- Gamboa J, Cortés M, Rodríguez A. Incidencia 
de Demodex folliculorum en blefaritis. Rev Mex Oftalmol 2003; 77 (2): 44-7. Disponible en: http://www.medigraphic. com/pdfs/revmexoft/rmo-2003/rmo032c.pdf [accedido el 18 de diciembre de 2011].

14.- Perdomo J, Palacios S, Acosta K, Carrero M, González E, Soler S. Evaluación de la efectividad del tratamiento homeopático en pacientes con diagnóstico clínico de blefaritis anterior. Rev Cubana Med Milit 2007; pp 36 (3). Disponible en: http://www.sld.cu/ galerias/pdf/sitios/mednat/evaluacion_de_la efectividad_del_tratamiento_homeopatico_ en_pacientes_con_diagnostico_clinico_de blefaritis_anterior.pdf [accedido el 18 de diciembre de 2011].

15.- Sanabria R, Arrúa M, Fariña N, Laspina F, Samudio M, Carpinelli, et al. P093. Demodex spp. en pacientes con blefaritis crónica del hospital de clínicas. Libro de resúmenes. XIX Congreso Latinoamericano de Parasitología. Asunción, Paraguay. 22, 23, 24 de octubre de 2009: pp 252. Disponible en: http://www.flap2009.com/files/block_8/ libroresumenesFLAP2009.pdf [accedido el 24 de diciembre de 2011].

16.- Rezusta A, Escobar S, Revillo M, Baldellou. Respuesta al tratamiento con metronidazol de un caso de infección dérmica por Demodex folliculorum. Posters Antiparasitarios. Poster AP-2. Pp 106. Disponible en: http:// seq.es/seq/0214-3429/16/supp1/105.pdf http://www.flap2009.com/files/block_8/ libroresumenesFLAP2009.pdf [accedido el 24 de diciembre de 2011].

17.- Aguilar A, Berra A. Blefaritis crónica. Discusión de caso. Archivos de Alergia e Inmunología Clínica 2001; 3 (3). Disponible en: http://www.archivos.alergia.org.ar/ material/32001/2001_03_06_discucion.pdf [Consultado el 14 de diciembre de 2011].

18.- Morfin B. Demodocidosis en una paciente tratada como blefaroconjuntivitis alérgica. Reporte de un caso. Revista Alergia México 2003; L (6): pp 232-6. Disponible en: http:// www.escuelaeims.com/pdf/investigacion/ demodicidosis.pdf [accedido el 20 de diciembre de 2011].

19.- Alonzo M, Guarneros A, Valdés R. Rosácea ocular: presentación de un caso. Rev Cent Dermatol Pascua 2006; 15 (3): 173-6. Disponible en: http://www.medigraphic.com/ pdfs/derma/cd-2006/cd063h.pdf [accedido el 17 de diciembre de 2011].

20.- Acariformes, Demodex spp. Disponible en: http://farmapuntes.wikispaces.com/file/view/ Tema $+8+-+$ Acariformes $\% 3 \mathrm{~B}+\operatorname{los}^{+} \% \mathrm{C} 3 \% \mathrm{~A}$ 1 caros $+\% 28$ Demodex, + Sarcoptes\%29.pdf. [accedido el 16 de diciembre de 2011]

21.- Rodríguez H. Prevalencia de Demodex spp. en pacientes con blefaritis. Anales de la Facultad de Medicina de la Universidad Nacional Mayor de
San Marcos 2000; 64 (4): 299-304. Disponible en: http://sisbib.unmsm.edu.pe/bvrevistas/ anales/v61_n4/prev_demo_pac.htm [accedido el 17 de diciembre de 2011].

22.- Gatta C, Comunale E, Menghi C. Infestación por Demodex spp.: un caso clínico. Rev Argentina Microbiol 2009; 41: 118. Disponible en: http://www.scielo.org.ar/pdf/ram/v41n2/ v41n2a12.pdf [accedido el 19 de diciembre de 2011].

23.- Nava A, Corredor R, Tovilla J, Muñoz S. Blefaritis por Demodex folliculorum. Rev Fac Med UNAM 2000; 43 (4): 125-9. Disponible en: http://www.ejournal.unam.mx/rfm/no43-4/ RFM43402.pdf [accedido el 20 de diciembre de 2011].

24.- Millás E. Parasitación por Demodex folliculorum. Control de calidad SEIMC. Disponible en: http://www.seimc.org/control/ revisiones/parasitologia/demodex.pdf [accedido el 19 de diciembre de 2011].

25.- Liu J, Sheha H, Tseng S C. Pathogenic role of Demodex mites in blepharitis. Curr Opin Allergy Clin Immunol. 2010; 10 (5): 505-10. Disponible en http://www.ncbi.nlm.nih.gov/ pubmed/20689407.

26.- Holzchuh F G, Hida R Y, Moscovici B K, Villa Albers M B, Santo R M, Kara-José N, et al. Clinical treatment of ocular Demodex folliculorum by systemic ivermectin. Am J Ophthalmol 2011; 151 (6): 1030-4. 